\title{
Menschen in außergewöhnlichen Umgebungen
}

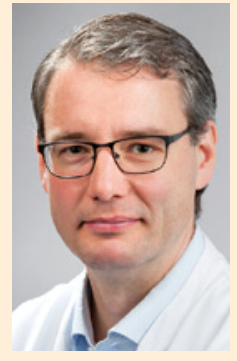

Liebe Kolleginnen und Kollegen,

in der Flug-, Tropen-/Reise-, Berg-/Expeditions-, Luft-/Raumfahrt- wie auch in der maritimen Medizin bezieht sich ein Großteil des ärztlichen Tätigkeitsfelds auf die Krankheitsprävention beziehungsweise Abwendung von gesundheitlichen Gefahren. Oftmals stehen uns dabei Menschen gegenüber, die einer beruflichen Tätigkeit in besonderen Umfeldern wie in den Tropen, auf dem Meer, in der Luft, im Weltall nachkommen wollen, was in der Regel eine besondere Fitness voraussetzt. Diese außergewöhnlichen Tätigkeitsfelder gehen oftmals auch mit besonderen Berufsbildern einher, denen sich diese Menschen voller Begeisterung hinwenden. Es ist daher auf unseren Fachgebieten erforderlich, entsprechende Tauglichkeitsuntersuchungen gewissenhaft und sorgfältig durchzuführen und über präventive Maßnahmen aufzuklären beziehungsweise diese zur Anwendung zu bringen. Dies geht aber nur dann, wenn wir Daten zu Erkrankungen aus den entsprechenden Tätigkeitsfeldern sammeln und auswerten.

Ein überraschender medizinischer Befund bei der arbeitsmedizinischen Untersuchung kann dabei nicht nur die weitere Ausübung der jeweiligen Erwerbstätigkeit gefährden, sie kann auch den Berufstraum erschüttern, wie Andreas Knöffler in seiner Kasuistik vor Augen führt (mit glücklicherweise gutem Ausgang). Das gleiche Thema wird unter einer völlig anderen Perspektive auch von den Kollegen Markus Stuhr et al. ab Seite 14 beschrieben: Die Gefahren, die mit der Errichtung von Windturbinen auf hoher See einhergehen, fasst ihr Artikel eindrucksvoll zusammen. Einen wiederum gänzlich verschiedenen Blickwinkel auf die beruflichen Risiken eines dieser besonderen Berufe zeigt der Beitrag von Torsten Pippig, der sich mit Wirbelsäulenverletzungen bei Luftwaffenangehörigen nach Verlassen des Kampfjets mit Hilfe des Schleudersitzes befasst. Burkhard Rieke widmet sich in seinem Artikel dem Thema Gelbfieberimpfung und kommentiert aus seiner Sicht die Bedeutung der von der WHO vor gut 1,5 Jahren ausgesprochenen Empfehlung zur einmal lebenslangen Impfung. An der hierzu bis dato anhaltenden Diskussion zeigt sich, dass sich die Empfehlungen der WHO zu Tropenkrankheiten beziehungsweise deren Prävention letztlich doch eher auf die Bedingungen und Hintergründe der endemischen Länder selbst beziehen und weniger auf die dorthin Reisenden. So ist es sicher wünschenswert, dass in Gelbfieberendemiegebieten beheimatete Menschen zumindest einmal im Leben geimpft werden. Bei dieser Empfehlung spielen sicherlich auch Überlegungen zu Praktikabilität, Kosten und Verfügbarkeit von Impfstoffen in den Endemiegebieten eine Rolle. In der Reisemedizin hingegen können wir uns eine individualisierte Präventivmedizin leisten, sodass zum Beispiel bei bestimmten Veränderungen des Immunsystems eine möglicherweise sinnvolle Wiederimpfung nach 10 Jahren weiterhin ihre Bedeutung haben kann. Der Artikel von Günther Slesak schließlich zeigt uns, wie initial rätselhafte Beschwerdebilder nach Besuch einer entlegenen Insel erst dadurch aufgeklärt werden können, dass wir uns in unseren Fachgebieten vernetzen, uns austauschen und miteinander kommunizieren. Auch dazu dient unser Fachjournal.

Mit dem Jahr 2014 ging ein ereignisreiches Jahr zu Ende. Die Aufmerksamkeit, die insbesondere die Tropen-/ Reisemedizin durch den Ebolaausbruch in Westafrika erhalten hat, sollte nun auch wieder dazu genutzt werden, den Blick auf die alltäglichen und ungelösten Probleme unseres Tätigkeitsfelds zu lenken. Ich hoffe, Sie haben beim Lesen dieser Ausgabe wieder viel Freude und gewinnen interessante Erkenntnisse. Das Jahr ist noch nicht zu weit fortgeschrittenen, um Ihnen ein erfolgreiches und interessantes Jahr $2015 \mathrm{zu}$ wünschen. 